\title{
Preclinical Evaluation of a P2X7 Receptor-Selective Radiotracer: PET Studies in a Rat Model with Local Overexpression of the Human P2X7 Receptor and in Nonhuman Primates
}

\author{
Dieter Ory*1,2, Sofie Celen*1, Rik Gijsbers ${ }^{2,3}$, Chris Van Den Haute ${ }^{3,4}$, Andrey Postnov ${ }^{5}$ Michel Koole $^{5}$, \\ Caroline Vandeputte ${ }^{5}$, José-Ignacio Andrés ${ }^{6}$, Jesus Alcazar ${ }^{6}$, Meri De Angelis ${ }^{6}$, Xavier Langlois ${ }^{6}$, \\ Anindya Bhattacharya ${ }^{6,7}$, Mark Schmidt ${ }^{8}$, Michael A. Letavic ${ }^{7}$, Wim Vanduffel ${ }^{9}$, Koen Van Laere ${ }^{5}$, Alfons Verbruggen ${ }^{1}$, \\ Zeger Debyser ${ }^{2}$, and Guy Bormans ${ }^{1}$ \\ ${ }^{I}$ Laboratory for Radiopharmacy, Department of Pharmaceutical and Pharmacological Sciences, KU Leuven, Leuven, Belgium; \\ ${ }^{2}$ Laboratory for Molecular Virology and Gene Therapy, Department of Pharmaceutical and Pharmacological Sciences, KU Leuven, \\ Leuven, Belgium; ${ }^{3}$ Leuven Viral Vector Core, KU Leuven, Leuven, Belgium; ${ }^{4}$ Laboratory for Neurobiology and Gene Therapy, \\ Department of Neurosciences, KU Leuven, Leuven, Belgium; ${ }^{5}$ Nuclear Medicine and Molecular Imaging, Department of Imaging and \\ Pathology, University Hospital and KU Leuven, Leuven, Belgium; ${ }^{6} J a n s s e n$ Research and Development, Discovery Sciences, a \\ Division of Janssen-Cilag, Toledo, Spain; ${ }^{7}$ Janssen Research and Development, LLC, San Diego, California; ${ }^{8}$ Janssen Research and \\ Development, Neuroscience Discovery, a Division of Janssen Pharmaceutica Nevada, Beerse, Belgium; and ${ }^{9}$ Laboratory for \\ Neuro- and Psychophysiology, Department of Neurosciences, KU Leuven, Leuven, Belgium
}

The P2X7 receptor (P2X7R) orchestrates neuroinflammation, and this is the basis for an increased interest in the development of antagonists inhibiting P2X7R function in the brain. This study provides the preclinical evaluation of ${ }^{11} \mathrm{C}-J \mathrm{NJ}-54173717$, a PET tracer for P2X7R in both rats and nonhuman primates. Methods: ${ }^{11} \mathrm{C}-\mathrm{JNJ}-54173717$ is a high-affinity radiotracer for the human P2X7R (hP2X7R). Biodistribution and radiometabolite studies were performed. Viral vectors encoding either enhanced green fluorescent protein-hP2X7R or 3flag-hP2X7R were engineered and validated in cell culture. hP2X7R was regionally overexpressed in the rat striatum after stereotactic injection of viral vectors. Dynamic small-animal PET studies were performed in vector-injected rats and in healthy monkeys using ${ }^{11} \mathrm{C}-J N J-54173717$. Results: The affinity of JNJ-54173717 was $1.6 \pm 0.1 \mathrm{nM}$ in a rat cortex P2X7R membrane binding assay. In a functional assay at the recombinant human and rat P2X7R orthologs, the half maximal inhibitory concentration $\left(\mathrm{IC}_{50}\right)$ of $\mathrm{JNJ}-54173717$ was $4.2 \pm 0.01 \mathrm{nM}$ and $7.6 \pm 0.01 \mathrm{nM}$, respectively. The rat biodistribution study showed that ${ }^{11} \mathrm{C}-\mathrm{JNJ}-54173717$ crossed the blood-brain barrier and was cleared from plasma mainly via the hepatobiliary pathway. A polar radiometabolite was found in rat plasma. No radiometabolites were detected in rat brain. Dynamic small-animal PET showed binding of ${ }^{11} \mathrm{C}-\mathrm{JNJ}-54173717$ in the striatum expressing hP2X7R, with rapid washout from the noninjected control striatum and other brain regions. Likewise, ${ }^{11} \mathrm{C}-J \mathrm{NJ}-54173717$ PET signal was blocked by a chemically distinct P2X7R ligand, indicating specific binding to P2X7R in the monkey brain. Conclusion: JNJ-54173717 is a high-affinity P2X7R antagonist. An animal rat model stably expressing hP2X7R was developed and validated, identifying favorable characteristics for

Received Nov. 19, 2015; revision accepted Apr. 5, 2016.

For correspondence or reprints contact: Guy Bormans, Laboratory for Radiopharmacy, Campus Gasthuisberg O\&N2, Herestraat 49 Box 821, BE-3000 Leuven, Belgium.

E-mail: Guy.Bormans@pharm.kuleuven.be

${ }^{*}$ Contributed equally to this work.

Published online May 19, 2016.

COPYRIGHT (c) 2016 by the Society of Nuclear Medicine and Molecular Imaging, Inc.
${ }^{11} \mathrm{C}-J N J-54173717$ as a PET radioligand for in vivo visualization of hP2X7R. ${ }^{11} \mathrm{C}-J N J-54173717$ selectively visualized P2X7R in the monkey brain, and this radioligand will be further evaluated in a clinical setting to study P2X7R expression levels in neurodegenerative disorders.

Key Words: P2X7 receptor; neuroinflammation; PET; viral vector; non-human primate

J Nucl Med 2016; 57:1436-1441

DOI: 10.2967/jnumed.115.169995

T ncreasing evidence suggests an active role of neuroinflammation in the progression of neurodegenerative disorders such as Alzheimer's disease and Parkinson's disease (1-3). Neuroinflammation is a well-orchestrated, dynamic, multicellular process. Microglial cells make up the innate immune system of the central nervous system and are key cellular mediators of neuroinflammatory processes. Upon neuronal injury or infection, they become activated, thereby releasing neurotoxic substances, amplifying neuroinflammation and leading to neurodegeneration (M1 phenotype). On the other hand, they also provide tissue repair by releasing antiinflammatory cytokines and neurotrophic factors (M2 phenotype). However, the current repertoire of activated microglia phenotypes and phenotype development might be more variable and complex (4). PET provides a sensitive noninvasive imaging technique to study and quantify receptor and enzyme expression. A radiolabeled tracer for a protein expressed during neuroinflammation and more specifically for overactivated microglia would allow in vivo detection and quantification of neuroinflammation in an early stage of the disease and could facilitate the development and evaluation of specific antineuroinflammatory treatment (5).

Until now, translocator protein (TSPO) was the most studied biomarker to image neuroinflammation using PET. However, recent 
findings indicate that TSPO polymorphism results in differences in binding affinity of PET radioligands for TSPO (6). As a consequence, knowledge of the binding characteristics of a PET ligand for TSPO of patients is required to correctly interpret PET images, complicating image analysis. In contrast to TSPO, more is known about the pharmacology of the $\mathrm{P} 2 \mathrm{X} 7$ receptor $(\mathrm{P} 2 \mathrm{X} 7 \mathrm{R})$ and its role during inflammation. The mechanism of action involves inflammasome activation and IL- $1 \beta$ release. Moreover, caspase- 1 activation, needed for IL-1 $\beta$ release, occurs within the NLRP3 inflammasome in response to an increased concentration of extracellular adenosine triphosphate and thus P2X7R activation. In turn, IL-1 $\beta$ is one of the proinflammatory cytokines that initiate the neuroinflammatory cascade $(7,8)$. In addition, several animal models for neurodegenerative disorders such as Alzheimer's disease and Huntington's disease (9-11) showed that proinflammatory cytokines correlated with upregulated P2X7R expression and increased adenosine triphosphate sensitivity, putting forward $\mathrm{P} 2 \mathrm{X} 7 \mathrm{R}$ as a valuable alternative marker for microglial activation and inflammation $(8,12)$. In a recent study, ${ }^{3} \mathrm{H}-\mathrm{A}-804598$ was used to determine the ex vivo brain P2X7R occupancy of different P2X7R antagonists, indicating that this class of molecules crosses the blood-brain barrier and supporting the feasibility of in vivo visualization of $\mathrm{P} 2 \mathrm{X} 7 \mathrm{R}$ with $\mathrm{PET}$ in the brain (13-15). An additional hurdle, however, is the P2X7R interspecies difference reported for several molecules, giving rise to different binding affinities for mouse, rat, and human P2X7R (hP2X7R) (16-18).

In this study, we developed a humanized animal model by stable expression of the $\mathrm{hP} 2 \mathrm{X} 7 \mathrm{R}$ in rat brain, which can be applied in the validation and evaluation of P2X7R PET tracers. To obtain efficient gene transfer, we applied viral vector technology, engineering a hP2X7R reporter system using HIV-based lentiviral vectors (LVs) for validation in cell culture settings, and recombinant adeno-associated viral (rAAV) vectors in the brain in vivo (19). JNJ-54173717 $(20,21)$, which has nanomolar affinity for rat P2X7R and hP2X7R, was radiolabeled with ${ }^{11} \mathrm{C}$ and evaluated in this rat model using small-animal PET. In contrast to several molecules described in the literature (1618), this compound shows little interspecies differences. This PET tracer was further validated in vivo with biodistribution studies and plasma and brain radiometabolite analysis after intravenous injection in rats and in vitro by performing autoradiography binding studies on normal rat brain slices, slices of a rat brain overexpressing hP2X7R in striatum, and wild-type (WT) and P2X7R knockout mouse brain slices. Finally, a monkey small-animal PET study was performed with ${ }^{11} \mathrm{C}-J \mathrm{NJ}-54173717$ in baseline and blocking conditions using 2 chemically distinct P2X7R selective ligands.

\section{MATERIALS AND METHODS}

The methods used for the in vitro pharmacology, radiosynthesis of the radioligand, biodistribution studies, viral vector construction and production, rat plasma and perfused rat brain radiometabolite analysis, cell culture and transduction, Western blot analysis, stereotactic injections, immunocytochemistry, cell uptake experiments, and in vitro autoradiography have been described in the supplemental materials (available at http://jnm.snmjournals.org). The research protocol was approved by the local Animal Ethics Committees and was performed according to guidelines of the European Ethics Committee.

\section{Small-Animal PET Imaging}

Imaging experiments were performed on a Focus 220 microPET scanner (Concorde Microsystems). Baseline scans were obtained in 3 rats at $10 \mathrm{wk}$ after viral vector injection. For a pretreatment experiment, the authentic reference JNJ54173717 was dissolved in a vehicle containing
$20 \%$ (2-hydroxypropyl)- $\beta$-cyclodextrine and 2 equivalents $\mathrm{HCl}$. This formulation was injected subcutaneously $45 \mathrm{~min}$ before tracer injection at a dose of $10 \mathrm{mg} / \mathrm{kg}(n=3$; pretreatment protocol based on ex vivo receptor occupancy study results).

Rats were injected with approximately $74 \mathrm{MBq}$ of ${ }^{11} \mathrm{C}-\mathrm{JNJ}-54173717$ via a tail vein under isoflurane anesthesia $(2.5 \%$ in oxygen at a flow rate of $1 \mathrm{~L} / \mathrm{min}$ ). List-mode dynamic 90-min small-animal PET scans were acquired. Acquisition data were Fourier-rebinned in 24 time frames $(4 \times$ $15,4 \times 60,5 \times 180,8 \times 300$, and $3 \times 600 \mathrm{~s}$ ) and reconstructed using maximum a posteriori iterative reconstruction. The images were spatially aligned to a rat brain ${ }^{18} \mathrm{~F}-\mathrm{FDG}$ template in Paxinos coordinates (22) using affine transformation, allowing the use of a predefined volumeof-interest map. Time-activity curves were generated for the striatum, cerebellum, and total brain for each individual scan using PMOD software (version 3.4; PMOD Technologies).

Kinetic modeling based on the simplified reference tissue model (SRTM) (23) was performed to quantify differences in radioactivity concentration between the left and right striatum. The left striatum was evaluated as a reference tissue.

\section{Small-Animal PET Study in Rhesus Monkeys}

Two juvenile male rhesus monkeys (Macaca mulatta, 5 and $6.8 \mathrm{~kg}$ ) were used in this small-animal PET study. Sedation was performed by an intramuscular injection of a combination of $0.3 \mathrm{~mL}$ of xylazine (Rompun [Bayer], 2\% solution) and $0.35 \mathrm{~mL}$ of ketamine (Nimatek [Eurovet], $100 \mathrm{mg} / \mathrm{mL}$ ). About $60 \mathrm{~min}$ after the first injection, the monkey received an additional dose of $0.15 \mathrm{~mL}$ of xylazine and $0.175 \mathrm{~mL}$ of ketamine via intravenous injection. During the last part of the scan, dosing was done less frequently, based on the heartbeat frequency. PET imaging was performed using a Focus 220 tomograph (Siemens/ Concorde Microsystems), which has a nominal transaxial resolution of $1.35 \mathrm{~mm}$ (full width at half maximum). The $\mathrm{O}_{2}$ and $\mathrm{CO}_{2}$ saturation in the blood and the heartbeat were constantly monitored. Temperature was regulated via a heating pad. The breathing frequency and the eye response were checked visually. The monkey was injected with \pm 185 $\mathrm{MBq}$ of ${ }^{11} \mathrm{C}-J \mathrm{NJ}-54173717$ via the vena saphena. For the self-block experiment, the authentic reference JNJ54173717 was dissolved in a vehicle containing $20 \%$ (2-hydroxypropyl)- $\beta$-cyclodextrine and 2 equivalents $\mathrm{HCl}$. In a second pretreatment experiment, JNJ-42253432 (a central nervous system-penetrant P2X7R antagonist and structurally unrelated compound (15)) was dissolved in a vehicle containing $20 \%$ (2-hydroxypropyl)- $\beta$-cyclodextrine and 0.75 equivalents $\mathrm{HCl}$ (no vehicle was injected in the baseline scan). These formulations were injected intravenously $10 \mathrm{~min}$ before tracer injection at a dose of $2.5 \mathrm{mg} / \mathrm{kg}$. Listmode dynamic 90-min small-animal PET scans were acquired. Acquisition data were Fourier-rebinned in 24 time frames $(4 \times 15,4 \times 60,5 \times$ $180,8 \times 300$, and $3 \times 600 \mathrm{~s}$ ) and reconstructed using maximum a posteriori iterative reconstruction. Data were analyzed using PMOD software, and volumes of interest were defined on the summed images and time-activity curves were drawn. During both baseline and pretreatment scans, blood samples were collected at 10, 30, and $60 \mathrm{~min}$ after tracer injection via the other vena saphena, this to determine radiometabolites of ${ }^{11} \mathrm{C}-\mathrm{JNJ}-54173717$ in plasma.

\section{RESULTS}

\section{In Vitro Pharmacology of JNJ-54173717}

The compound displayed high potency in a functional assay. The half maximal inhibitory concentration $\left(\mathrm{IC}_{50}\right)$ of JNJ-54173717 was $4.2 \pm 0.01 \mathrm{nM}$ and $7.6 \pm 0.01 \mathrm{nM}$, at the human and rat P2X7R orthologs, respectively. Consistent with the functional inhibition, JNJ-54173717 displayed high-affinity binding at the native P2X7R channels in the rat cortex. The affinity of the compound was $1.6 \pm$ $0.1 \mathrm{nM}$ at the rat brain P2X7R channel (Fig. 1). 


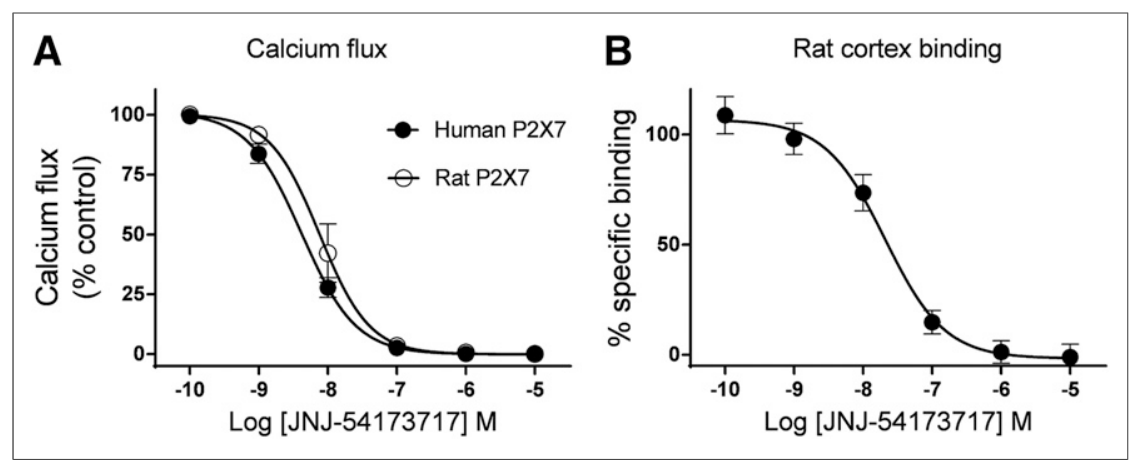

FIGURE 1. In vitro pharmacology of JNJ-54173717. (A) IC $\mathrm{I}_{50}$ of $\mathrm{JNJ}-54173717$ was $4.2 \pm 0.01 \mathrm{nM}$ and $7.6 \pm 0.01 \mathrm{nM}$, at the human and rat P2X7 orthologs, respectively. (B) Affinity of compound was $1.6 \pm 0.1 \mathrm{nM}$ at the native P2X7 channels in rat cortex. Data are expressed as mean \pm SEM from 3 independent experiments in duplicate (each symbol is a mean of 6 data points).
The reconstructed radiochromatograms from perfused rat cerebellum and cerebrum HPLC analysis at $30 \mathrm{~min}$ after tracer injection showed only 1 radioactive peak corresponding to intact ${ }^{11} \mathrm{C}-\mathrm{JNJ}-54173717$ eluting at $9 \mathrm{~min}(n=2$; chromatograms not shown). The fraction of both more polar and more lipophilic radiometabolites was negligible $(<2 \%)$. The recovery of the HPLC (XBridge) column--injected radioactivity was $85 \% \pm 6 \%$.

\section{Evaluation of ${ }^{11} \mathrm{C}-\mathrm{JNJ}-54173717$ in hP2X7R Cell Model: Validation in Cell Culture by Western Blot Analysis, Immunocytochemistry, and Cell Uptake Experiments}

SHSY5Y (human dopaminergic neuroblastoma) cell lines stably expressing $\mathrm{hP} 2 \mathrm{X} 7 \mathrm{R}$ were generated via transduction with HIV-based LVs (LV_eGFP-hP2X7R and LV_3flag-hP2X7R) encoding human P2X7R N-terminally fused to eGFP or a 3 flag tag driven from a ubiquitous human cytomegalovirus immediate-early promoter (eGFP is enhanced green fluorescent protein) (Supplemental Figs. 1A and 1D).

Expression of full-length P2X7R was confirmed by Western blot analysis for SHSY5Y cells transduced with LV_eGFP-hP2X7R and LV_3flag-hP2X7R, showing a band of $100 \mathrm{kDa}$ corresponding to the eGFP-tagged $\mathrm{hP} 2 \mathrm{X} 7 \mathrm{R}$ protein and a band of $80 \mathrm{kDa}$ corresponding to the 3flag tagged hP2X7R (Supplemental Figs. 1B and $1 \mathrm{E}$, respectively). In parallel, we verified subcellular distribution of the tagged hP2X7R by immunocytochemistry (Supplemental Figs. 1C and $1 \mathrm{~F}$ ). Both protein fusions were primarily located at the plasma membrane, indicating that the tagging did not affect the subcellular distribution. No immunoreactivity was seen in nontransduced SHSY5Y cells. As a proof of principle, we verified ${ }^{11} \mathrm{C}-\mathrm{JNJ}-54173717$ uptake for SHSY5Y cells overexpressing hP2X7R. The percentage of total radioactivity in the cell fraction per milligram of protein was calculated for LV_eGFP-hP2X7R, LV_eGFP, LV_3flag-hP2X7R, LV_3flag-eGFP, and nontransduced SHSY5Y cells. The ratio of total uptake of radioactivity in cells transduced with LV_eGFP-hP2X7R to cells transduced with LV_eGFP was $5.1 \pm 0.6$. The ratio of total

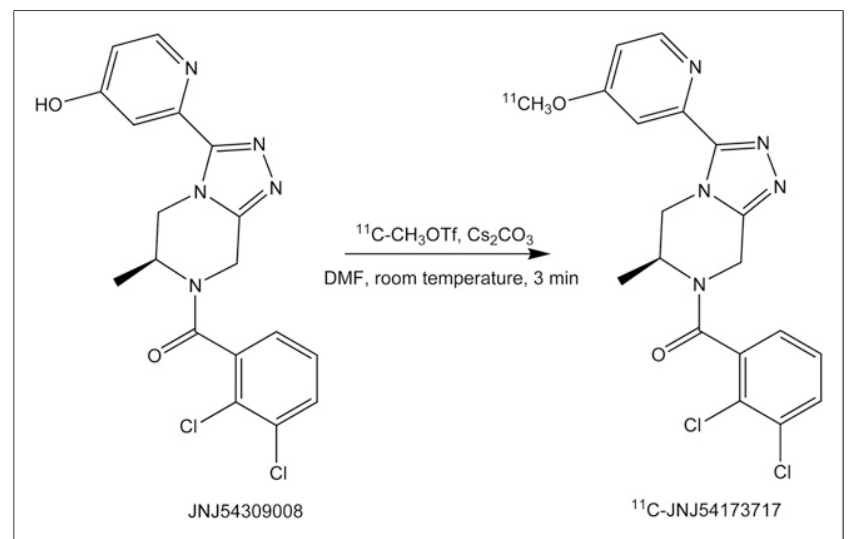

FIGURE 2. Radiosynthesis of ${ }^{11} \mathrm{C}-J \mathrm{NJ}-54173717 .{ }^{11} \mathrm{C}$-methyl triflate $\left({ }^{11} \mathrm{C}-\mathrm{MeOTf}\right)$ was bubbled with a flow of helium through a solution of precursor $(0.2 \mathrm{mg})$ and $\mathrm{Cs}_{2} \mathrm{CO}_{3}(1-3 \mathrm{mg})$ in anhydrous dimethyl formamide (DMF; $0.2 \mathrm{~mL}$ ). Reaction mixture was left at room temperature for $3 \mathrm{~min}$ and purified with reversed-phase high-pressure liquid chromatography. 
activity bound by LV_3flag-hP2X7R cells to total activity bound to cells transduced with LV_3flag-eGFP was $3.6 \pm 0.4$.

Evaluation of ${ }^{11} \mathrm{C}-J N J-54173717$ in hP2X7R Locoregional Transgenic Rat Model: Validation of rAAV_3flag-hP2X7R by In Vitro Autoradiography, Small-Animal PET, and Histology

In Vitro Autoradiography. Higher tracer binding was observed in WT mouse brain than in P2X7R knockout mouse brain (ratio of WT total binding to knockout total binding $=3.3$ ), though the latter showed some specific binding (Supplemental Figs. 2A-2D; ratio of knockout total binding to knockout nonspecific binding $=2.0$; ratio of WT total binding to WT nonspecific binding $=8.9 ; n=4$ ). The in vitro binding study on the transversal rat brain sections of vector-injected rats showed higher tracer binding in the rAAV_3flag-hP2X7R injected striatum (right striatum) than in the rAAV_3flag-eGFP-injected striatum (left striatum) (Fig. 3A; Supplemental Fig. 3). The right-to-left activity ratio was $3.1 \pm 0.9$ $(n=4)$. Tracer binding could be blocked using $10 \mu \mathrm{M}$ A-740003 (Fig. 3B). Supplemental Figures $3 \mathrm{G}$ and $3 \mathrm{H}$ present the results of the in vitro autoradiography study on brain sections of WT rats $(n=3)$. Highest binding is observed to white matter, which can also be blocked with $10 \mu \mathrm{M}$ A-740003. Displaceable binding is also observed to the colliculus, cortex, and striatum.

Small-Animal PET Imaging of $h P 2 X 7 R$ Expression. A highintensity signal was observed in the $\mathrm{hP} 2 \mathrm{X} 7 \mathrm{R}$ vector-injected right striatum compared with only background radioactivity in the eGFP control vector injected (Fig. 4A). This was also reflected in the baseline time-activity curves in which accumulation of radioactivity was observed in the right striatum with washout from the left striatum (Fig. 4C). The average binding potential $( \pm \mathrm{SD})$ calculated with SRTM was $0.63 \pm 0.16(n=3)$. Pretreatment by subcutaneous injection of JNJ-54173717 (10 mg/kg) at $45 \mathrm{~min}$ before tracer injection resulted in a significant decrease of the radioactivity concentration in the hP2X7R-expressing right striatum $(n=3)$ (Figs. 4B and 4D).

Histology. To corroborate expression of the viral vectors, 3 animals were sacrificed after the small-animal PET scan to perform histology. The expression of the viral vectors was in accordance with the radioactivity accumulation observed in the small-animal PET data ( $n=3$; Supplemental Fig. 4).

\section{Small-Animal PET Study in Rhesus Monkeys}

A relatively high initial brain uptake of ${ }^{11} \mathrm{C}-\mathrm{JNJ}-54173717$ was observed in the monkey small-animal PET study (SUV, 3.3). The uptake of ${ }^{11} \mathrm{C}-\mathrm{JNJ}-54173717$ was homogeneous throughout the monkey brain. Reduced uptake of ${ }^{11} \mathrm{C}-\mathrm{JNJ}-54173717$ was observed after pretreatment $(2.5 \mathrm{mg} / \mathrm{kg}$ intravenously, $10 \mathrm{~min}$ before tracer

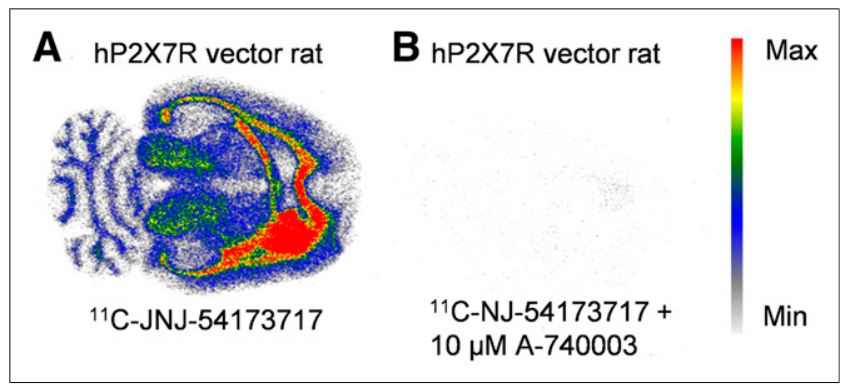

FIGURE 3. In vitro autoradiography binding studies on slices of rat brain injected with $\mathrm{hP} 2 \mathrm{X} 7 \mathrm{R}$ vector using ${ }^{11} \mathrm{C}-\mathrm{JNJ}-54173717(n=4) .{ }^{11} \mathrm{C}$ JNJ-54173717 shows specific binding to rAAV_3flag-hP2X7R-injected site (A), which can be blocked with $10 \mu \mathrm{M} \mathrm{A-740003} \mathrm{(B).} \mathrm{Max}=$ maximum; $\min =$ minimum.

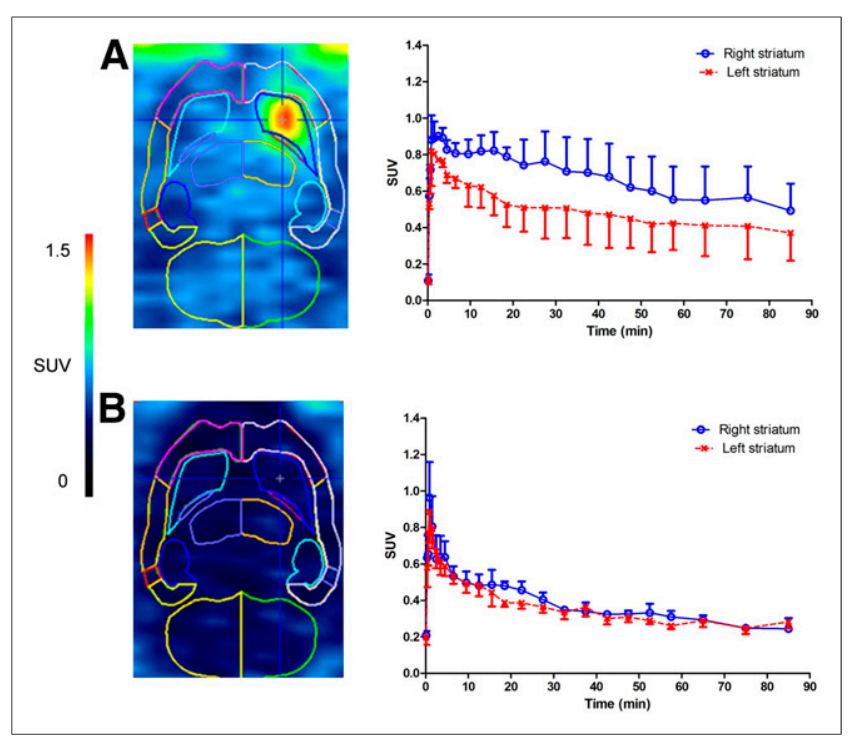

FIGURE 4. Small-animal PET data for ${ }^{11} \mathrm{C}-J N J-54173717$ in $\mathrm{hP} 2 \mathrm{X} 7 \mathrm{R}$ rat model (right striatum, rAAV_3flag-hP2X7R, and left striatum, rAAV_3flagGFP). Representative averaged small-animal PET images (same animal) of whole scan $(90 \mathrm{~min})$ were acquired at baseline with corresponding time-activity curves $(\mathrm{A} ; n=3)$ and after pretreatment with authentic reference material with corresponding time-activity curves $(\mathrm{B} ; n=3)$. Data were normalized for injected radioactivity and body weight of animal (SUV). Error bars correspond to SD.

injection) with cold JNJ-54173717 and with JNJ-42253432 (Fig. 5) (15). The plasma SUVs of intact ${ }^{11} \mathrm{C}-\mathrm{JNJ}-54173717$ for the first baseline scan were $0.55,0.33$, and 0.24 , and for pretreatment (with JNJ-54173717) they were $0.52,0.34$, and 0.25 at 10,30 , and $60 \mathrm{~min}$ after tracer injection, respectively. The plasma SUVs of intact ${ }^{11} \mathrm{C}$ JNJ-54173717 for the second baseline scan in a different monkey were $0.69,0.39$, and 0.26 , and for pretreatment (with JNJ42253432) they were $0.54,0.37$, and 0.27 at 10,30 , and $60 \mathrm{~min}$ after tracer injection, respectively.

\section{DISCUSSION}

The P2X7R plays an important role during neuroinflammation and therefore synthesis of small molecules that can act as antagonists, inhibiting P2X7R function in the brain, are of particular interest. The interspecies difference of P2X7R may, however, be a major hurdle in the development of P2X7-targeted drugs. Most P2X7R antagonists described in the literature are not active or weakly active against $\mathrm{P} 2 \mathrm{X} 7 \mathrm{R}$ isoforms present in mouse and rat, underscoring the need of an animal model expressing the hP2X7R. During the last decade, PET has become a valuable tool in the development of new drugs. Noninvasive imaging of P2X7R using PET would allow studying this receptor in both healthy and diseased conditions. Furthermore, it would be useful in the drug development process because it gives direct insight in the relation between P2X7R occupancy and the dose of the candidate antagonist, allowing the validation or invalidation of a new drug candidate at an early stage, thereby saving a lot of effort and money, making the drug development process more efficient.

In this study, we therefore aimed to develop a cell and animal model expressing the human isoform of the P2X7R and validate these humanized models with a newly developed high-affinity P2X7R PET tracer.

Unlike ${ }^{11} \mathrm{C}-\mathrm{A}-740003$, a P2X7R-selective tracer developed by Janssen et al. (24), ${ }^{11} \mathrm{C}-\mathrm{JNJ}-54173717$ was able to cross the 


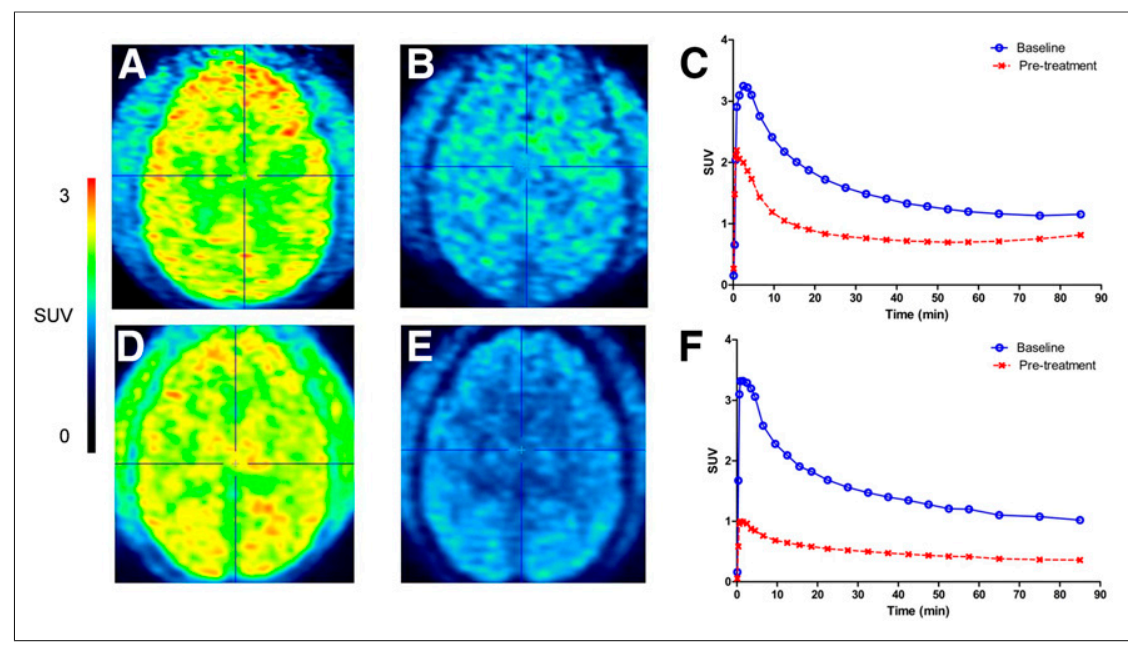

FIGURE 5. ${ }^{11} \mathrm{C}-J N J-54173717$ PET in rhesus monkey. Time-weighted averaged small-animal PET images (10-30 $\mathrm{min}$ ) of baseline study $(\mathrm{A})$ and after pretreatment with authentic reference material ( $2.5 \mathrm{mg} / \mathrm{kg}$ intravenously $10 \mathrm{~min}$ before tracer injection) (B). (C) Corresponding total brain timeactivity curves at baseline and after pretreatment. Time-weighted averaged small-animal PET images (10-30 min) of second PET scan in different monkey at baseline (D) and after pretreatment with JNJ-42253432 (2.5 mg/kg intravenously $10 \mathrm{~min}$ before tracer injection) (E). (F) Corresponding total brain time-activity curves at baseline and after pretreatment.

blood-brain barrier as was shown in biodistribution studies in normal rats. Excretion was mainly hepatobiliary, as was expected in view of the lipophilicity of the tracer. To use a PET radioligand for accurate measurements of target density and target occupancy in drug dose occupancy studies, knowledge of radiometabolites is required, more particularly to which extent radiometabolites are present in plasma and their ability to pass the blood-brain barrier and contribute to specific or nonspecific binding. Therefore, the in vivo stability of ${ }^{11} \mathrm{C}-\mathrm{JNJ}-54173717$ was examined in plasma and the perfused brain of normal rats. In plasma, 1 unidentified polar radiometabolite was detected, probably generated by cleavage of the amide bond. The radiotracer appeared to be, however, quite stable because still $70 \%$ of the radioactivity in plasma was in the form of intact tracer $30 \mathrm{~min}$ after radiotracer injection. In the brain, ideally, no radiometabolites should be present, because they may complicate PET quantification. A negligible fraction of radiometabolites $(<2 \%)$ of ${ }^{11} \mathrm{C}-\mathrm{JNJ}-54173717$ was found in the cerebrum and cerebellum $30 \mathrm{~min}$ after injection.

In vitro autoradiography studies with ${ }^{11} \mathrm{C}-J N J-54173717$ performed on rat brain slices showed higher tracer binding to the $\mathrm{hP} 2 \mathrm{X7R}$ viral vector-injected striatum than the contralateral eGFP viral vector-injected striatum, proving the specificity of tracer binding to hP2X7R. Interestingly, in vitro autoradiography binding studies on WT mouse brain slices showed specific binding, suggesting this tracer has also affinity for the mouse P2X7R. In WT rat brain, high displaceable binding to white matter was observed. This binding pattern was, however, not observed in the binding study with $3 \mathrm{H}-\mathrm{A}$ 804598 performed by Able et al. (13) nor in the binding studies with ${ }^{11} \mathrm{C}-J \mathrm{NJ}-54173717$ on the mouse brain sections.

Further evaluation and validation of our model and tracer was done using in vivo small-animal PET studies. Time-activity curves showed ${ }^{11} \mathrm{C}-\mathrm{JNJ}-54173717$ binding in the hP2X7R vector-injected striatum and fast washout from the contralateral eGFP vectorinjected striatum. Pretreatment experiments with the authentic reference material showed that tracer binding is specific. Binding potential values were calculated using the SRTM with the contralateral left striatum as a reference region. The obtained binding potential values were not that high. This can be explained by the fact that the transfected brain volume is much smaller than the striatum itself. Nevertheless, the use of mirror brain volume of interest is optimal for SRTM kinetic modeling, and the fits were robust. Because there is also endogenous expression of rat P2X7R throughout the rat brain (also in the contralateral striatum), this measures the increase in binding potential mediated by the expression of the hP2X7R. Confirmation of the expression of the injected viral vectors was done by histology on rat brain sections showing clear expression of the corresponding proteins.

In the monkey brain, pretreatment with the cold JNJ-54173717 and with the P2X7R antagonist JNJ-42253432 (15) reduced uptake of ${ }^{11} \mathrm{C}-J N J-54173717$, suggesting P2X7Rselective binding of ${ }^{11} \mathrm{C}-\mathrm{JNJ}-54173717$ in the rhesus monkey. Tracer binding in surrounding tissue was also lower after pretreatment, suggesting that P2X7R also blocks tracer retention in extracerebral tissues in-line with the ubiquitous expression of P2X7. The increase of tracer concentration at the end of the scan may indicate accumulation of radiometabolites in the brain. This was, however, not observed in the second study in a different monkey (baseline and pretreatment with JNJ-42253432) nor was it observed in rat studies. Almost no difference was observed in the metabolism of ${ }^{11} \mathrm{C}-\mathrm{JNJ}-54173717$ between the baseline and pretreatment study, indicating absence of inhibition of ${ }^{11} \mathrm{C}-\mathrm{JNJ}-54173717$ metabolism by authentic JNJ-54173717 or by JNJ-42253432.

\section{CONCLUSION}

We have developed a new PET brain reporter gene system based on hP2X7R expression via viral vector technology, which was validated with a new selective P2X7R PET tracer, ${ }^{11} \mathrm{C}-\mathrm{JNJ}-$ 54173717. This humanized animal model can be used in the development and screening of new drugs targeting the hP2X7R. ${ }^{11} \mathrm{C}-J N J-54173717$ selectively visualized P2X7R in the monkey brain, and this radioligand will be further evaluated in a clinical setting to study P2X7R expression levels in neurodegenerative disorders. We expect that also in humans, endogenous expression will be high enough to be quantified by ${ }^{11} \mathrm{C}-J N J-54173717$ PET, and application of the pseudoreference tissue model (25) may be a valid approach for quantification.

\section{DISCLOSURE}

The costs of publication of this article were defrayed in part by the payment of page charges. Therefore, and solely to indicate this fact, this article is hereby marked "advertisement" in accordance with 18 USC section 1734. Dieter Ory is a fellow of the Research Foundation Flanders. Koen Van Laere is a Senior Research Fellow for the Research Foundation Flanders. Viral vectors were engineered and produced by the Leuven Viral Vector Core (Leuven, Belgium). No other potential conflict of interest relevant to this article was reported. 


\section{ACKNOWLEDGMENTS}

We thank Ann Van Santvoort, Julie Cornelis, and Ivan Sannen for their assistance in the animal work.

\section{REFERENCES}

1. Glass CK, Saijo K, Winner B, Marchetto MC, Gage FH. Mechanisms underlying inflammation in neurodegeneration. Cell. 2010;140:918-934.

2. Chen M-K, Guilarte TR. Translocator protein $18 \mathrm{kDa}$ (TSPO): molecular sensor of brain injury and repair. Pharmacol Ther. 2008;118:1-17.

3. Jacobs AH, Tavitian B. Noninvasive molecular imaging of neuroinflammation. J Cereb Blood Flow Metab. 2012;32:1393-1415.

4. Eggen BJL, Raj D, Hanisch U-K, Boddeke HWGM. Microglial phenotype and adaptation. J Neuroimmune Pharmacol. 2013;8:807-823.

5. Rudin M, Weissleder R. Molecular imaging in drug discovery and development. Nat Rev Drug Discov. 2003;2:123-131.

6. Owen DR, Yeo AJ, Gunn RN, et al. An 18-kDa translocator protein (TSPO) polymorphism explains differences in binding affinity of the PET radioligand PBR28. J Cereb Blood Flow Metab. 2012;32:1-5.

7. Ohara K, Shimizu K, Matsuura S, et al. Toll-like receptor 4 signaling in trigeminal ganglion neurons contributes tongue-referred pain associated with tooth pulp inflammation. J Neuroinflammation. 2013;10:139-149.

8. Skaper SD, Debetto P, Giusti P. The P2X7 purinergic receptor: from physiology to neurological disorders. FASEB J. 2010;24:337-345.

9. Parvathenani LK, Tertyshnikova S, Greco CR, Roberts SB, Robertson B, Posmantur R. P2X7 mediates superoxide production in primary microglia and is up-regulated in a transgenic mouse model of Alzheimer's disease. J Biol Chem. 2003;278:13309-13317.

10. Sanz JM, Chiozzi P, Ferrari D, et al. Activation of microglia by amyloid $\beta$ requires P2X7 receptor expression. J Immunol. 2009;182:4378-4385.

11. Takenouchi T, Sekiyama K, Sekigawa A, et al. $\mathrm{P} 2 \mathrm{X} 7$ receptor signaling pathway as a therapeutic target for neurodegenerative diseases. Arch Immunol Ther Exp (Warsz). 2010;58:91-96.

12. Ory D, Celen S, Verbruggen A, Bormans G. PET radioligands for in vivo visualization of neuroinflammation. Curr Pharm Des. 2014;20:5897-5913.
13. Able SL, Fish RL, Bye H, et al. Receptor localization, native tissue binding and ex vivo occupancy for centrally penetrant $\mathrm{P} 2 \mathrm{X} 7$ antagonists in the rat. Br J Pharmacol. 2011;162:405-414.

14. Bhattacharya A, Wang Q, Ao H, et al. Pharmacological characterization of a novel centrally permeable P2X7 receptor antagonist: JNJ-47965567. Br J Pharmacol. 2013;170:624-640.

15. Lord B, Aluisio L, Shoblock JR, et al. Pharmacology of a novel central nervous system-penetrant P2X7 antagonist JNJ-42253432. J Pharmacol Exp Ther. 2014;351:628-641.

16. Donnelly-Roberts DL, Jarvis MF. Discovery of P2X7 receptor-selective antagonists offers new insights into P2X7 receptor function and indicates a role in chronic pain states. Br J Pharmacol. 2007;151:571-579.

17. Gunosewoyo H, Coster MJ, Kassiou M. Molecular probes for P2X7 receptor studies. Curr Med Chem. 2007;14:1505-1523.

18. Donnelly-Roberts DL, Namovic MT, Surber B, et al. [ $\left.{ }^{3} \mathrm{H}\right] \mathrm{A}-804598$ ([ $\left.{ }^{3} \mathrm{H}\right] 2$-cyano1-[(1S)-1-phenylethyl]-3-quinolin-5-ylguanidine) is a novel, potent, and selective antagonist radioligand for P2X7 receptors. Neuropharmacology. 2009;56:223-229.

19. Van der Perren A, Toelen J, Carlon M, et al. Efficient and stable transduction of dopaminergic neurons in rat substantia nigra by rAAV 2/1, 2/2, 2/5, 2/6.2, 2/7, 2/8 and 2/9. Gene Ther. 2011;18:517-527.

20. Savall BM, Wu D, De Angelis M, et al. Synthesis, SAR, and pharmacological characterization of brain penetrant $\mathrm{P} 2 \mathrm{X} 7$ receptor antagonists. ACS Med Chem Lett. 2015;6:671-676.

21. Rudolph DA, Alcazar J, Ameriks MK, et al. Novel methyl substituted 1-(5,6dihydro-[1,2,4]triazolo[4,3-a]pyrazin-7(8H)-yl)methanones are $\mathrm{P} 2 \mathrm{X} 7$ antagonists. Bioorg Med Chem Lett. 2015;25:3157-3163.

22. Casteels C, Vermaelen P, Nuyts J, et al. Construction and evaluation of multitracer small-animal PET probabilistic atlases for voxel-based functional mapping of the rat brain. J Nucl Med. 2006;47:1858-1866.

23. Lammertsma AA, Hume SP. Simplified reference tissue model for PET receptor studies. Neuroimage. 1996;4:153-158.

24. Janssen B, Vugts DJ, Funke U, et al. Synthesis and initial preclinical evaluation of the P2X7 receptor antagonist $\left[{ }^{11} \mathrm{C}\right] \mathrm{A}-740003$ as a novel tracer of neuroinflammation. J Labelled Comp Radiopharm. 2014;57:509-516.

25. Salinas CA, Searle GE, Gunn RN. The simplified reference tissue model: model assumption violations and their impact on binding potential. J Cereb Blood Flow Metab. 2015;35:304-311. 